\title{
Mechanical Decoupling Algorithm Applied to Electric Drive Test Bed
}

\author{
Song Qiang and Luo Lin \\ Beijing Institute of Technology, Beijing 10081, China \\ Correspondence should be addressed to Song Qiang; songqiang.xs@gmail.com
}

Received 14 March 2014; Accepted 17 June 2014; Published 17 August 2014

Academic Editor: Jingjing Zhou

Copyright (C) 2014 S. Qiang and L. Lin. This is an open access article distributed under the Creative Commons Attribution License, which permits unrestricted use, distribution, and reproduction in any medium, provided the original work is properly cited.

New approach and analysis are proposed in this paper to enhance the steady and rapidity of the electric drive test bed. Based on a basic drive motor dynamometer system (DMDS) test bed, detailed mathematical model and process control are established and analyzed. Relative gain array (RGA) method and diagonal matrix method are used to analyze the mechanical coupling caused by mechanical connection on the DMDS test bed, and the structure and algorithm of dynamic decoupling are proposed. Simulation and experiment all indicate that the designed decoupling method can efficiently improve the control accuracy and response speed.

\section{Introduction}

A number of electric drive systems are applied into industry field due to the rapid development of power electronic technology and AC electromotor control technology. In addition, because of the energy crisis, electric drive is more and more being taken into account for its pure using of electricity. With the increasing use of electric or generate motor, motor performance tests on test bed are becoming more and more universalizing for production permit. What is more, some predevelopment researches should be down on test bed with the virtual mechanical load attached to the electric drive system. Therefore, laboratories and research institutes are swarmed with test bed experiments.

In order to reduce risks, quality assurance of produce for manufactory is important. For instance, if electric vehicle wants to be legally put into production, regular test report of the drive motor performances, like efficiency map, released from some accredited testing organization is necessary. For every new products and sample tests, a lot of work should be finished on test bed. Nowadays, many test equipment has been provided with automatic test and storage function. However, for some steady test, such as efficiency or temperature rise of electromotor, testers will either waste much time on waiting steady state during the test or process plenty of data which including much meaningless ones after the test, and all of that is due to the bad control accuracy of motor controller or dynamic regulation of the test bed.

For test bed designing and improving, Works are mainly focused on hardware implement and software control. CAN bus or Profibus-DP communication is used in the test bed system to enhance the system integration and universality $[1,2]$. In order to improve the expandability and realtime performance, DSP or PLC is widely applied $[3,4]$. Ac electrical dynamometer is very popular in the electric drive test bed system, because it has a quick response and safety protection mechanism except its electricity feedback and bidirectional loading ability [5].

A few researchers study control algorithms in order to improve the whole DMDS system's steady and rapidity. Literature $[6,7]$ proposed an advanced control algorithm for load simulation test bed using a feed-forward compensator and a feedback controller. Although that system could enhance the control system accuracy, it was completely bounded to the load simulation application and was hard to apply to other types of test bench. Disturbance observer was developed to enhance the robustness of the test bed system [8], but it did not solve the essential problem for a generalized test bed system. In secondary regulation loading system, decoupling control algorithms are adopted to improve the loading accuracy $[9,10]$. In [9], the author used single neural PID control algorithm to realize the decoupling design for 


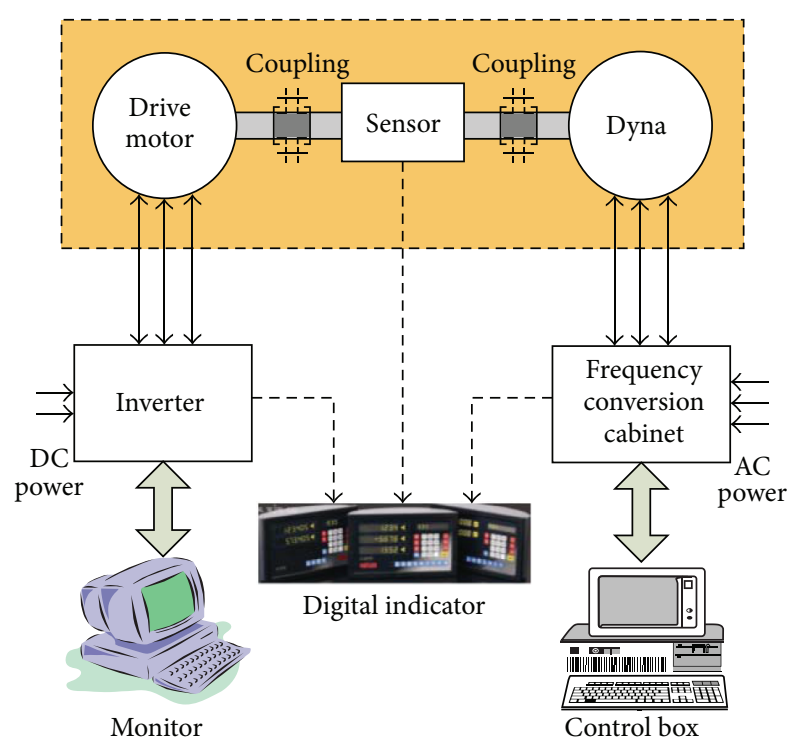

Figure 1: Basic structure of the test bed system.

a hydraulic loading system. However, its complex calculation was not conducive to practical use. In contrast, [10] used diagonal matrix theory to realize the decoupling easily.

Actually, for electric drive test bed, similar situation is encountered as well as secondary regulation loading system. There is no research focusing on the mechanical coupling of the electric drive test bed. The connected drive motor and dynamometer have a typical interaction during their independent control. This paper focuses on this problem and tries to analyze and design an algorithm using MIMO techniques [11] to improve the system steady and rapidity of the electric drive test bed.

\section{Modeling of Test Bed}

According to the tested object, such as the electromotor itself, the transmission, or other mechanical parts and control algorithms, all kinds of electric drive test beds can be quite different. Here some researches are down based on a traditional and simplest test bed system. Figure 1 is the structure of the system. It consists of driving motor system, dynamometer system, torque-speed transducer, an industrial personal computer (IPC), and other necessaries. The transducer, drive motor, and dynamometer are connected by two rubber resilient couplings here.

In fact, electromotor may be a complex system due to its various control algorithms and working environment. Hence, it is hard to finish a complete electromotor model. However some mainly factors can be used to describe the dynamic characteristics of the test bed, such as inertia and friction coefficient.

For the test bed system, components are always changing because of the uncertain tested objects except for dynamometer system. Therefore, the drive motor system and dynamometer system are taken as independent control system so as to improve the universality here. The transducer

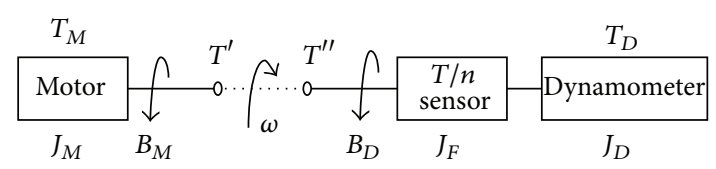

Figure 2: Physical model of the test bed.

and couplings can be classified to the dynamometer part. Then two mathematical models can be worked out based on the above assumption. Figure 2 is the physical model of the research object.

$T_{M}$ and $T_{D}$ are electromagnetic torque of the drive motor and dynamometer, respectively. $J_{m}, J_{d}$, and $J_{N}$ are inertias which belong to drive motor and dynamometer and gearbox, respectively. $B_{M}$ and $B_{D}$ are coefficients concerning resisting torque of drive motor and dynamometer caused by viscous friction damping or wind resistance. $T^{\prime}$ and $T^{\prime \prime}$ are action and reaction torque right after the drive motor output shaft.

In order to further improve the universality of the model, here the motor electromagnetic torque, load of the motor, and disturbance force of the test bench are used as vectors, which means signed arithmetic is effective. This is because those forces have nothing to do with the rotation of the test bed shaft and can be either positive or negative relative to the rotation direction. However, kinds of resisting torques may have a specific reversed direction to the rotation of the test bed.

According to the dynamic character of rotation part, the dynamic equation of the drive motor side can be written as follows:

$$
T_{M}+T^{\prime}-B_{M}(\omega)=J_{M} \frac{d \omega}{d t}
$$

Simultaneously, for the dynamometer, the dynamic equation can be written as follows:

$$
\begin{gathered}
T^{\prime \prime}+T_{D}+T_{F}-B_{D}(\omega)=\left(J_{D}+J_{N}\right) \frac{d \omega}{d t} \\
J_{\mathrm{DN}}=J_{D}+J_{N} .
\end{gathered}
$$

\section{Control Analysis}

The system's resisting torque is actually a strong nonlinear factor. But, on the condition of not highly accurate system, it can be supposed to be a linear function of the angular speed [12]. If so, the transfer function from torque to speed of the electromotor can be easily written out as follows:

$$
\begin{gathered}
G_{M E}=\frac{\omega}{\left(T_{M}+T^{\prime}\right)} \\
G_{D E}=\frac{\omega}{\left(T^{\prime \prime}+T_{D}+T_{F}\right)},
\end{gathered}
$$

where $G_{M E}$ and $G_{D E}$ are the transfer functions of the drive motor and dynamometer, respectively. If $T^{\prime}$ and $T^{\prime \prime}$ are considered as scalar, then their relationship can be obtained as $T^{\prime}=-T^{\prime \prime}$. 


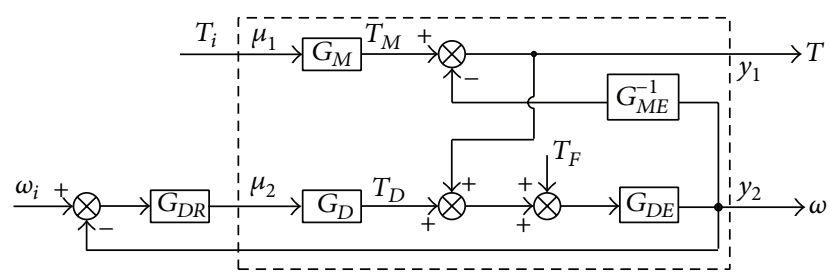

FIgURE 3: Dynamic block diagram for the test bench.

The mechanical torque produced by drive motor and the speed controlled by dynamometer are the really important things that what the testers care about during the test procedure. We can put $T^{\prime \prime}$ and $\omega$ left beside the equal sign in (3) for the convenience of analysis:

$$
\begin{gathered}
T^{\prime \prime}=T_{M}-\frac{\omega}{G_{M E}} \\
\omega=\left(T^{\prime \prime}+T_{D}+T_{F}\right) \cdot G_{D E} .
\end{gathered}
$$

Equation (4) is the physical model of the test bed, which does not include the control system of the electromotor. Considering the complexity of the control algorithm of the electromotor, an abstract transfer function can be used here to describe the control system. Figure 3 is the dynamic block diagram of the test bed system.

Where $G_{M}$ and $G_{D}$ are the control algorithms of drive motor and dynamometer, like vector control, direct torque control. $T$ is the mechanical torque of drive motor, which is equal to $T^{\prime \prime} . T_{i}$ and $\omega_{i}$ are the desired torque and speed command for drive motor and dynamometer, separately. $T_{D R i}$ is the actual regulatory variable after regulator in the control algorithm of dynamometer. $G_{D R}$ is the regulator independent of the dynamometer control algorithm.

Seen from Figure 3, the speed regulator of the dynamometer control algorithm is extracted out. It is useful to do this on the following researches. In fact, the torque and rotate speed of the test bed is expected to be changed quickly and accurately while working conditions or instructions varied. However, Figure 3 tells that the drive motor and the dynamometer have an obvious interaction, which is completely caused by the physical connection between the two motors. Actually, this feature is called V-norm coupling, which has a typical characteristic of cross repeated influence among each control channel $[13,14]$. When the mechanical torque of the drive motor has been changed, it will affect the speed condition of the dynamometer, and in turn, speed change will influence the mechanical torque through transfer function $G_{M E}$. Thus, interaction transmits on and on if system goes without damping part. An ideal test bed is that control channel between drive motor and dynamometer would not affect each other.

For coupling, there have been many methods to study it. Here, RGA method is utilized to further confirm and analyze the coupling. Static relative gain is the ratio of the first amplification coefficient to the second amplification coefficient of the researched system. Similar method can be used to calculate the dynamic relative gain here [15].
Without regarding the regulator, taking the dotted part in Figure 3 as the studied object, and transfer functions referred from $y_{1}, y_{2}$ to $\mu_{1}, \mu_{2}$ can be worked out as follows:

$$
\begin{aligned}
& y_{1}=\frac{\mu_{1} G_{M}-\mu_{2} G_{D} G_{D E} G_{M E}^{-1}}{1+G_{D E} G_{M E}^{-1}} \\
& y_{2}=\frac{\mu_{1} G_{M} G_{D E}+\mu_{2} G_{D} G_{D E}}{1+G_{D E} G_{M E}^{-1}},
\end{aligned}
$$

where $y_{1}, y_{2}, \mu_{1}$, and $\mu_{2}$ are the variables to replace the corresponding physical parameters in Figure 3. First of all, relative gain from $\mu_{1}$ to $y_{1}$ can be obtained from (5):

$$
\lambda_{11}=\frac{1}{\left(1+G_{D E} G_{M E}^{-1}\right)} .
$$

Because the sum of every row or column of the RGA is equal to 1 , the complete RGA can be got as follows:

$$
\Lambda=\left[\begin{array}{ll}
\lambda_{11} & \lambda_{12} \\
\lambda_{21} & \lambda_{22}
\end{array}\right]=\left[\begin{array}{cc}
\frac{1}{1+G_{D E} G_{M E}^{-1}} & \frac{G_{D E} G_{M E}^{-1}}{1+G_{D E} G_{M E}^{-1}} \\
\frac{G_{D E} G_{M E}^{-1}}{1+G_{D E} G_{M E}^{-1}} & \frac{1}{1+G_{D E} G_{M E}^{-1}}
\end{array}\right] .
$$

RGA method indicates that if the matrix is not an identity one, coupling will certainly exist [15]. We can look back to see the relative gain coefficient $\lambda_{11}$. After inserting the expressions of $G_{D E}$ and $G_{M E}$, detailed relative gain can be got:

$$
\lambda_{11}=\frac{1}{1+\left(\left(J_{M} s+B_{M}\right) /\left(J_{D} s+B_{D}\right)\right)} .
$$

$J_{M}, B_{M}, J_{D}$, and $B_{D}$ are the physical parameters, so $\lambda_{11}$ could not be one, and therefore, expression (7) would not be an identity matrix, too. However, from the structure of expression (8), we can see that $\lambda_{11}$ is approaching to one while dynamometer parameters are becoming larger and drive motor parameters are becoming smaller. That is to say, the larger the ratio between dynamometer and drive motor parameters is, the steadier and more accurate the system will be.

\section{Mechanical Decoupling Design}

Though mechanical coupling could not be avoided, software decoupling control algorithm can be adopted to solve or weaken the coupling. Literature [13] has discussed many decoupling methods based on different types of coupling systems. It points out that if different control specification is applied, decoupling part will be relative simple. What is more, if the decoupling part is placed after the system regulator, it will not contain any element of the system regulator which may does not have a definition structure. Literature [16] also pointed out that it was better not to bring in too much part of the original system into the decoupling part; otherwise system will loss stability. P-norm type of decoupling part, located right after the system regulator, is applied here. Figure 4 is the structure of P-norm decoupling links for the whole system. 


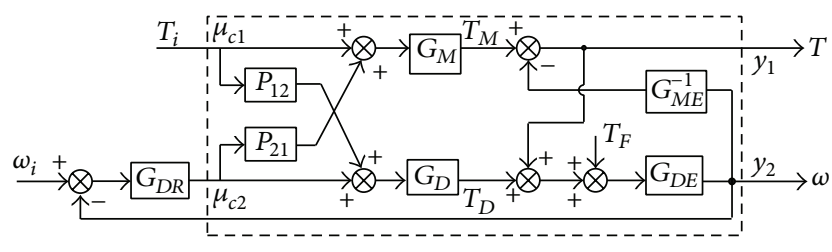

Figure 4: Decoupling design of the system.

Similar to the calculation process of (5), new transfer function can be got from Figure 4:

$$
\begin{aligned}
{\left[\begin{array}{l}
y_{1} \\
y_{2}
\end{array}\right]=} & {\left[\begin{array}{ll}
\frac{G_{M}-G_{D} G_{D E} G_{M E}^{-1} P_{12}}{1+G_{D E} G_{M E}^{-1}} & \frac{G_{M} P_{21}-G_{D} G_{D E} G_{M E}^{-1}}{1+G_{D E} G_{M E}^{-1}} \\
\frac{G_{M} G_{D E}+G_{D} G_{D E} P_{12}}{1+G_{D E} G_{M E}^{-1}} & \frac{G_{M} G_{D E} P_{21}+G_{D} G_{D E}}{1+G_{D E} G_{M E}^{-1}}
\end{array}\right] } \\
& \times\left[\begin{array}{l}
\mu_{c 1} \\
\mu_{c 2}
\end{array}\right],
\end{aligned}
$$

where $P_{12}$ and $P_{21}$ are the designed P-norm decoupling elements. $\mu_{c 1}$ and $\mu_{c 2}$ are new operating variables before the decoupling units.

Decoupling control theory also indicates that the premise of no coupling is that the system transfer function is also an identity array. So, to decouple the mechanical system, the transfer function array in expression (9) must be a diagonal matrix. Then $P_{12}$ and $P_{21}$ can be deduced out:

$$
\begin{gathered}
P_{12}=-\frac{G_{M}}{G_{D}} \\
P_{21}=\frac{G_{D}}{G_{M}} G_{D E} G_{M E}^{-1} .
\end{gathered}
$$

As stated above, $G_{M}$ and $G_{D}$ are the virtual transfer functions of the control algorithm of electromotor. Their specific expressions are quite hard to be written out. However, for a well debugged algorithm, $G_{M}$ and $G_{D}$ can nearly have a similar response characteristic. To simplify the decoupling part, we can ignore the differences between $G_{M}$ and $G_{D}$, and they can be eliminated:

$$
\begin{gathered}
P_{12}=-1 \\
P_{21}=G_{D E} G_{M E}^{-1} .
\end{gathered}
$$

After inserting definite parameters into (11), decoupling units can be finally written as follows:

$$
P_{12}=-1, \quad P_{21}=\frac{J_{M} s+B_{M}}{\left(J_{F}+J_{D}\right) s+B_{D}} .
$$

$P_{12}$ and $P_{21}$ are simple proportional component and lead-lag component. Both of them are easy to be physically implemented. In fact, on the occasion of relatively small inertia and low precision, unidirectional decoupling with $P_{12}$ can also generate a good effect.

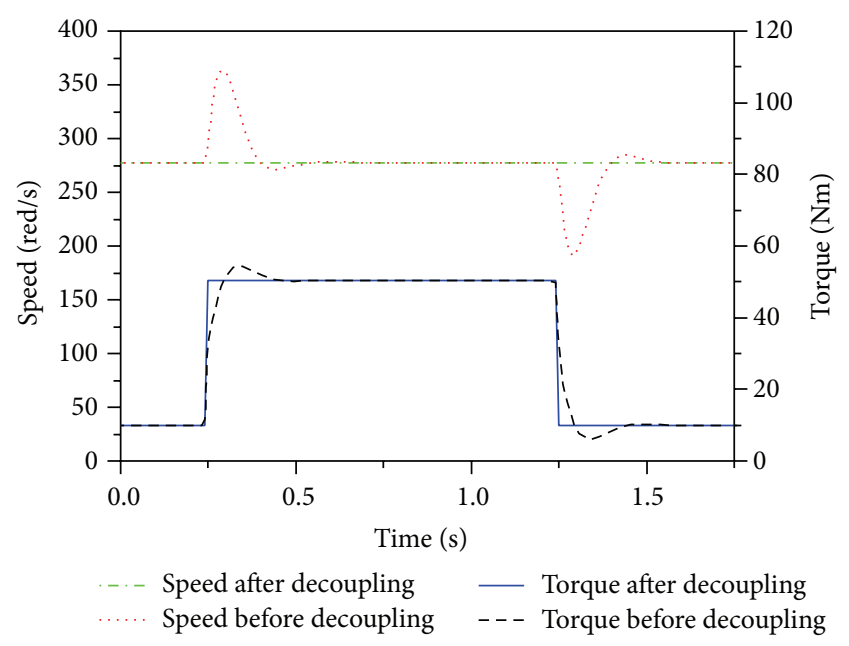

FIGURE 5: Speed response based on torque step input.

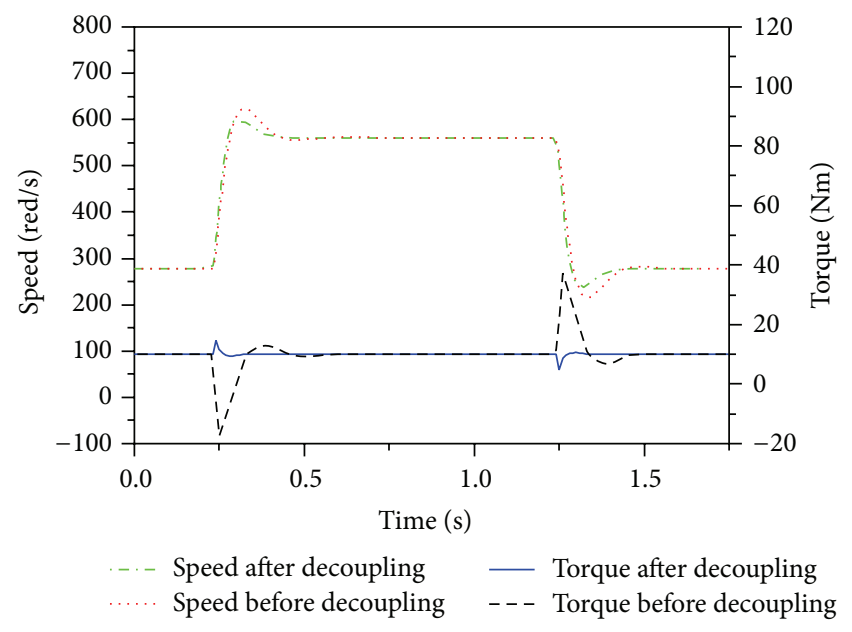

FIGURE 6: Torque response based on speed step input.

\section{Simulation and Experiment}

Referring to the above modeling and control analysis, simulation model can be set up using MATLAB/Simulink. Considering that the control algorithms of electromotor $G_{M}$ and $G_{D}$ have been simplified, look-up table method is adopted to finish the electromotor modeling, and it is assumed that they work well without time delay. Additionally, a traditional PI controller is used as the regulator of the dynamometer closeloop system. Simulations are finished based on responses of step inputs. Figures 5 and 6 are the simulation results.

Figure 5 is the speed response of the dynamometer under a drive motor's step torque input. Figure 6 is the torque response of the drive motor under a dynamometer's step torque input. Firstly, no matter dynamometer or drive motor, the control accuracy and response speed are all improved after decoupling implement seen from Figures 5 and 6. This illustrates that the typical characteristic of $\mathrm{V}$-norm coupling has a repeated cross influence between these two control channels. For instance, Figure 6 mainly studies the speed 
TABLE 1: Main parameters of the test bench.

\begin{tabular}{lccc}
\hline Name & Unit & Part & Value \\
\hline Peak power & $\mathrm{kW}$ & & $11 / 11$ \\
Peak torque & $\mathrm{Nm}$ & & $52 / 52$ \\
Peak speed & $\mathrm{r} / \mathrm{min}$ & Dynamometer/drive motor & $2000 / 2000$ \\
Inertia & $\mathrm{Kg} \cdot \mathrm{m}^{2}$ & & $0.042 / 0.042$ \\
Damping & 1 & & $0.005 / 0.003$ \\
coefficient & 1 & Transducer & 0.013 \\
Inertia & $\mathrm{kg} \cdot \mathrm{m}^{2}$ & &
\end{tabular}

response under torque step input, but at the condition of speed control being improved, torque response becomes more rapid and precise as well. Secondly, torque response in Figure 6 is not as good as speed response in Figure 5. On the one side, this may be due to the differential decoupling part which is easily to amplify small interference in the system, and on the other side, the torque-controlled channel does not have a close-loop feedback, which has no ability to resist external disturbance. It tells that it must be cautious to introduce differential part into the control system.

Actually, results in Figures 5 and 6 are based on precise mathematical model; so it may display a relative ideal control effect. On real test bed, there are more external disturbances and meanwhile accurate test bed parameters are hard to be obtained; so it would not as perfect as the simulation result. Based on similar control algorithm, a real test bed is built up using Simens S120 servo systems. Table 1 shows the main parameters of the test bed.

Figure 7 shows a simple experiment using a unidirectional decoupling with $P_{12}$.

Figure 7(a) features the result before decoupling, and Figure 7(b) features the decoupling one. Before and after decoupling, Figure 7 tells that peak-peak values of the dynamometer's response to step torque from up to down caused by drive motor are $211 \mathrm{r} / \mathrm{min}$ and $32 \mathrm{r} / \mathrm{min}$, respectively. In addition, average regulation times of dynamometer during a single step torque are $415 \mathrm{~ms}$ and $13 \mathrm{~ms}$. Through the analysis of the experimental data, it is obvious that system overshoot and regulation time have been improved greatly after applying the decoupling design. If the research is done on a larger test bench, coupling influence will be quite significant, and thus complete decoupling will be necessary for high-precision requirement.

\section{Conclusions}

This paper describes a detailed electric drive test bed, and a specific mathematical modeling, analysis, simulation, and experiment based on a DMDS are finished. Relative gain array method and diagonal matrix method are used to solve the mechanical decoupling between speed-controlled dynamometer and torque-controlled drive motor. From the analysis of the mathematical model, we can find that coupling between torque-controlled drive motor and speed-controlled dynamometer is inevitable due to the mechanical connection and the nonignorable test bed parameters, and what is

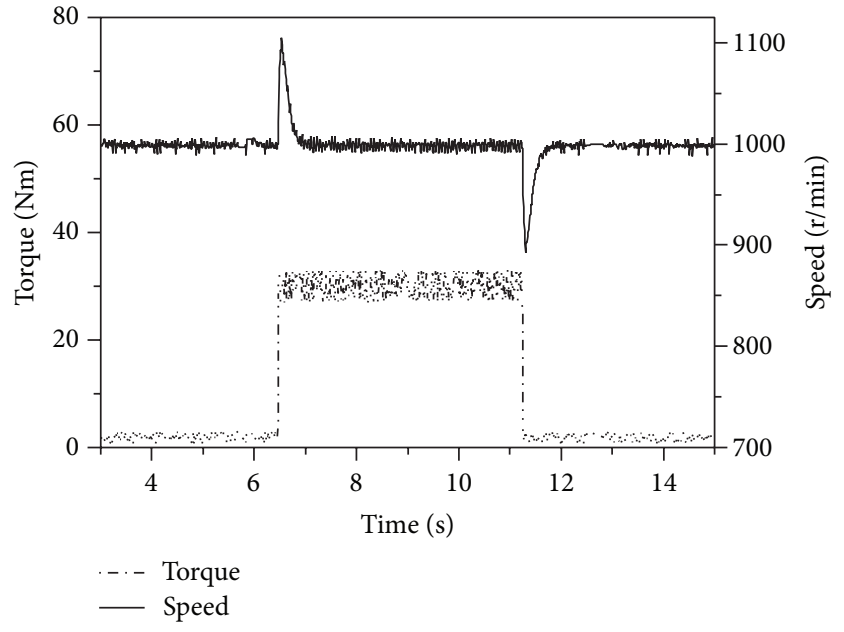

(a)

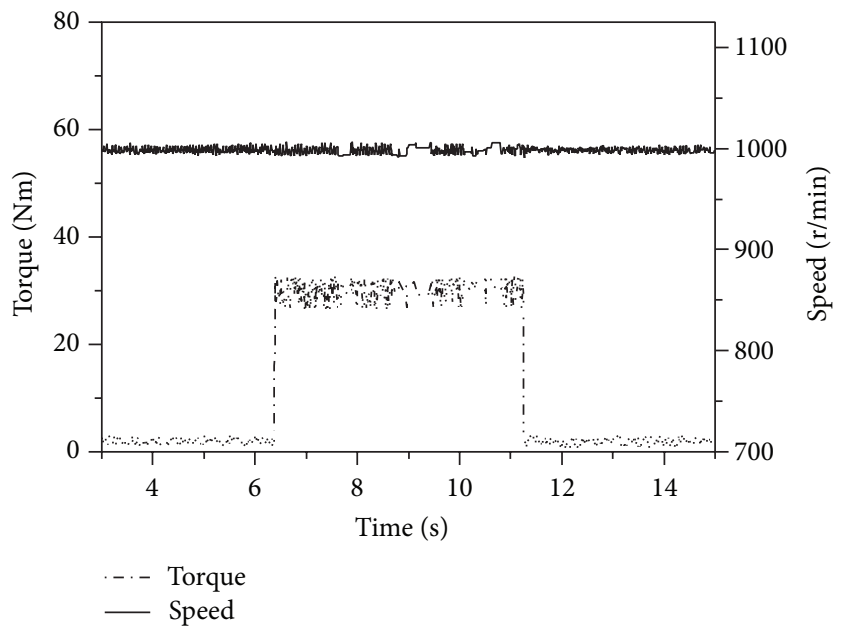

(b)

FIGURE 7: Speed response of dynamometer under step torque.

worse is that it is a V-norm coupling, which repeatedly interacts among torque and speed when changes happen. In addition, it tends to be more serious while the values of dynamometer's inertia and resisting torque become smaller than these parameters of drive motor.

To improve the test efficiency, a P-norm decoupling control program is presented out. The simulation and experiment strongly evidence that both speed control and torque control are highly improved through inserting decoupling compensation links after the system regulator. According to the specific application environments and tangible needs, complete decoupling with $P_{12}$ and $P_{21}$ or unidirectional decoupling with $P_{12}$ can be adopted. The presented method can be not only applied on the test system, but also used on the other purposes, such as load simulation system or hardwarein-loop system. 


\section{Conflict of Interests}

The authors declare that there is no conflict of interests regarding the publication of this paper.

\section{Acknowledgment}

This work was financially supported by International Science \& Technology Cooperation Program of China (Grant no. 2014DFG70840).

\section{References}

[1] G. S. Wu, L. Ling, and S. X. Fang, "Application of CAN bus of automatic measurement and control system in engine test-bed," Instrument Technique and Sensor, no. 12, pp. 79-80, 2007.

[2] N. Ge, G. Peng, L. Yu, and H. Liu, "Gearbox loading test bench based on DC speed regulation," Transaction of Beijing Institute of Technology, vol. 28, no. 1, pp. 70-74, 2008.

[3] D. Montesinos, S. Galceran, A. Sudria, and O. Gomis, "A laboratory test bed for PM brushless motor control," in Proceedings of the 11th IEEE Conference Power Electronics and Applications, p. 6, Dresden, Germany, 2005.

[4] R. B. Chen, M. R. Fei, H. H. Deng, and Y. Z. Huang, "The testbed of multi-intelligent control algorithms based on DSP," in Proceedings of the International Conference on Machine Learning and Cybernetics, vol. 2, pp. 952-957, Beijing, China, November 2002.

[5] J. Q. Yang and J. Huang, "Control and implementation of alternating current variable-frequency power dynamometer," Journal of Zhejiang University, vol. 40, no. 4, pp. 619-628, 2006.

[6] M. Rodič, K. Jezernik, and M. Trlep, "Dynamic emulation of mechanical loads: an advanced approach," IEE Proceedings: Electric Power Applications, vol. 153, no. 2, pp. 159-166, 2006.

[7] J. Arellano-Padilla, G. M. Asher, and M. Sumner, "Control of an AC dynamometer for dynamic emulation of mechanical loads with stiff and flexible shafts," IEEE Transactions on Industrial Electronics, vol. 53, no. 4, pp. 1250-1260, 2006.

[8] J. Back, K. Lee, I. Choy, J. Park, and H. Kim, "Emulation of dynamic mechanical load for dynamometer using disturbance observer based robust controller," in Proceedings of the 11th International Conference on Control, Automation and Systems (ICCAS '11), pp. 1037-1040, Gyeonggi-do, Korea, October 2011.

[9] S. Peng, Q. Ding, L. Qiu, Z. Wang, and Z. Pei, "Decoupling control of the secondary regulation load simulation test equipment for hydraulic chassis of engineering machinery vehicle," China Mechanical Engineering, vol. 17, no. 8, pp. 870-874, 2006.

[10] L. F. Tian, Z. C. Zhong, S. Y. Li, and Q. H. Liu, "Decoupling of hydraulic loading teststand with seeondary regulation," China Mechanical Engineering, vol. 8, no. 6, pp. 101-104, 1997.

[11] Q. Xu, "Design and development of a flexure-based dual-stage nanopositioning system with minimum interference behavior," IEEE Transactions on Automation Science and Engineering, vol. 9, no. 3, pp. 554-563, 2012.

[12] M. Rodič, K. Jezernik, and M. Trlep, "Control design in mechatronic systems using dynamic emulation of mechanical loads," in Proceedings of the IEEE International Symposium on Industrial Electronics (ISIE '05), pp. 1635-1640, Dubrovnik, Croatia, June 2005.

[13] C. H. Liu, "Multivariable coupling system description and Several general principles of the decoupling design," in Decoupling
Theory of Multivariable Process Control System, pp. 13-20, 121122, Beijing, China, 1st edition, 1984.

[14] Y. M. Li, J. M. Huang, and H. Tang, "A compliant parallel XY micromotion stage with complete kinematic decoupling," IEEE Transactions on Automation Science and Engineering, vol. 9, no. 3, pp. 538-553, 2012.

[15] D. X. Huang, J. C. Wang, and Y. H. Jin, "Decoupling control system," in Process Control System, pp. 233-235, Beijing, China, 1st edition, 2012.

[16] L. Menini, A. Tornambè, and G. Viola, "Input-output decoupling for m-inputs m-outputs linear mechanical systems through interconnection," in Proceedings of the 44th IEEE Conference on Decision and Control, and the European Control Conference (CDC-ECC '05), pp. 4590-4595, December 2005. 


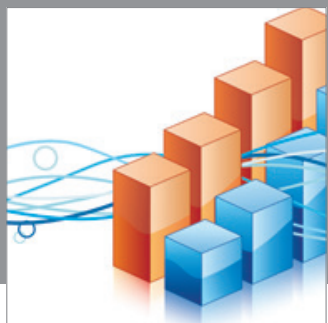

Advances in

Operations Research

mansans

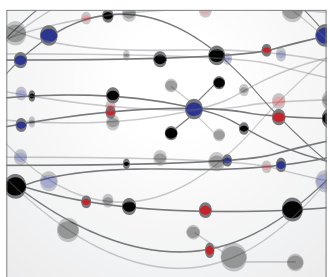

The Scientific World Journal
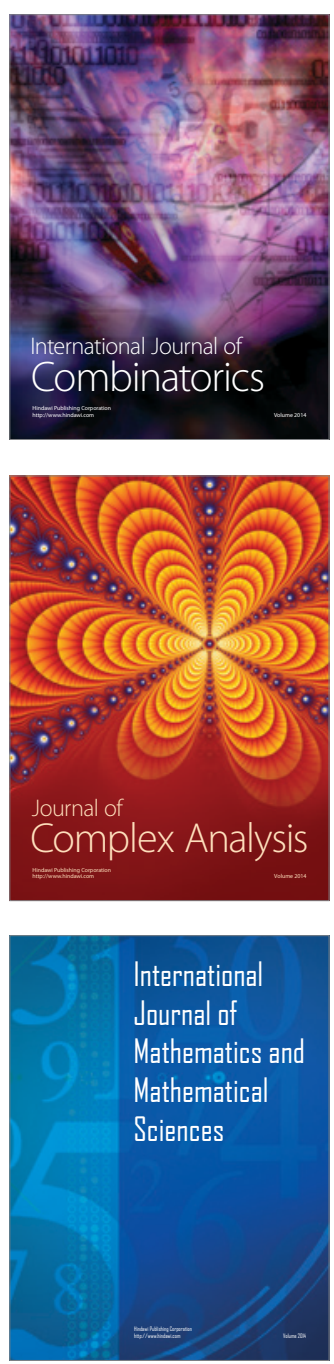
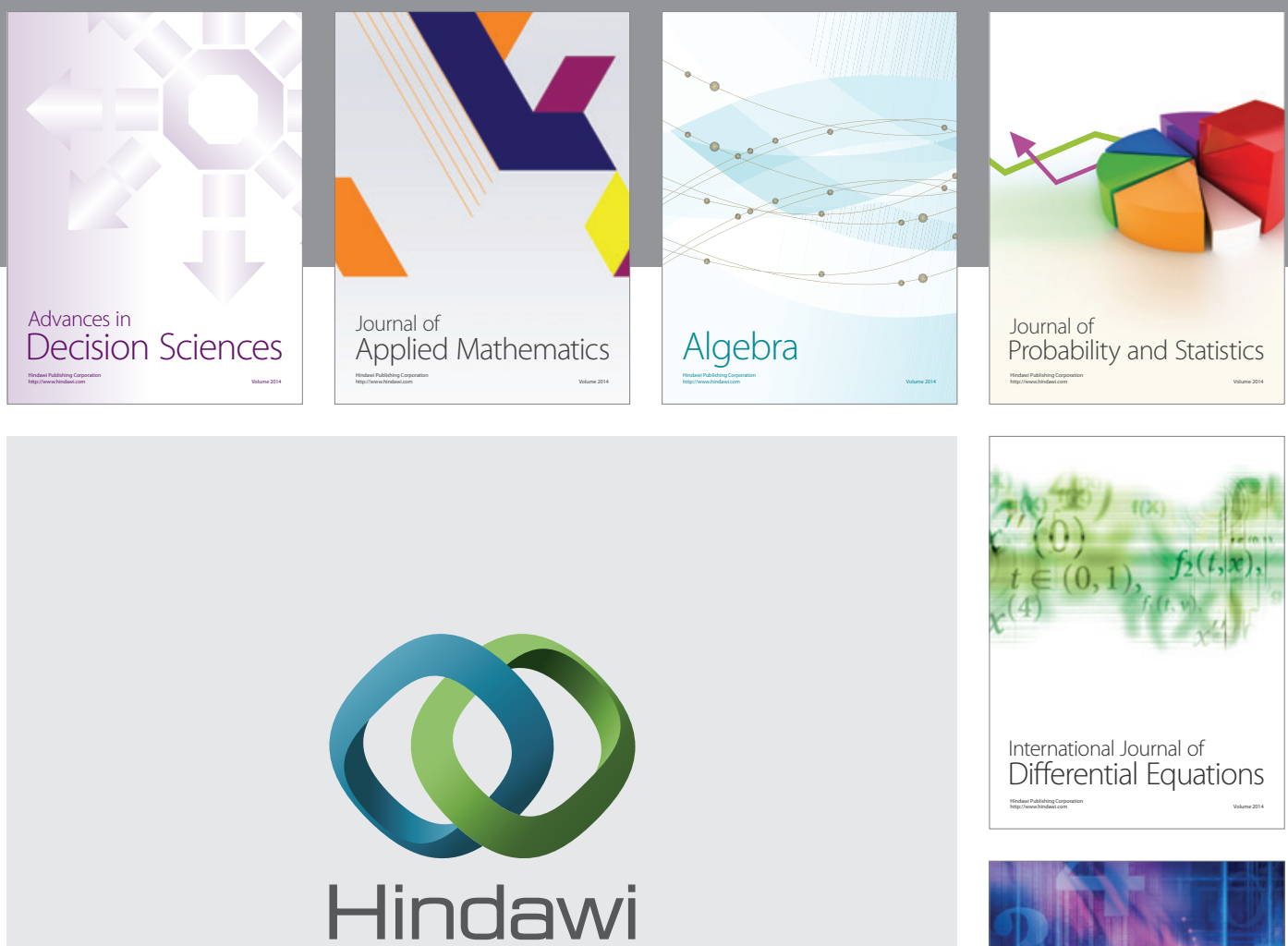

Submit your manuscripts at http://www.hindawi.com
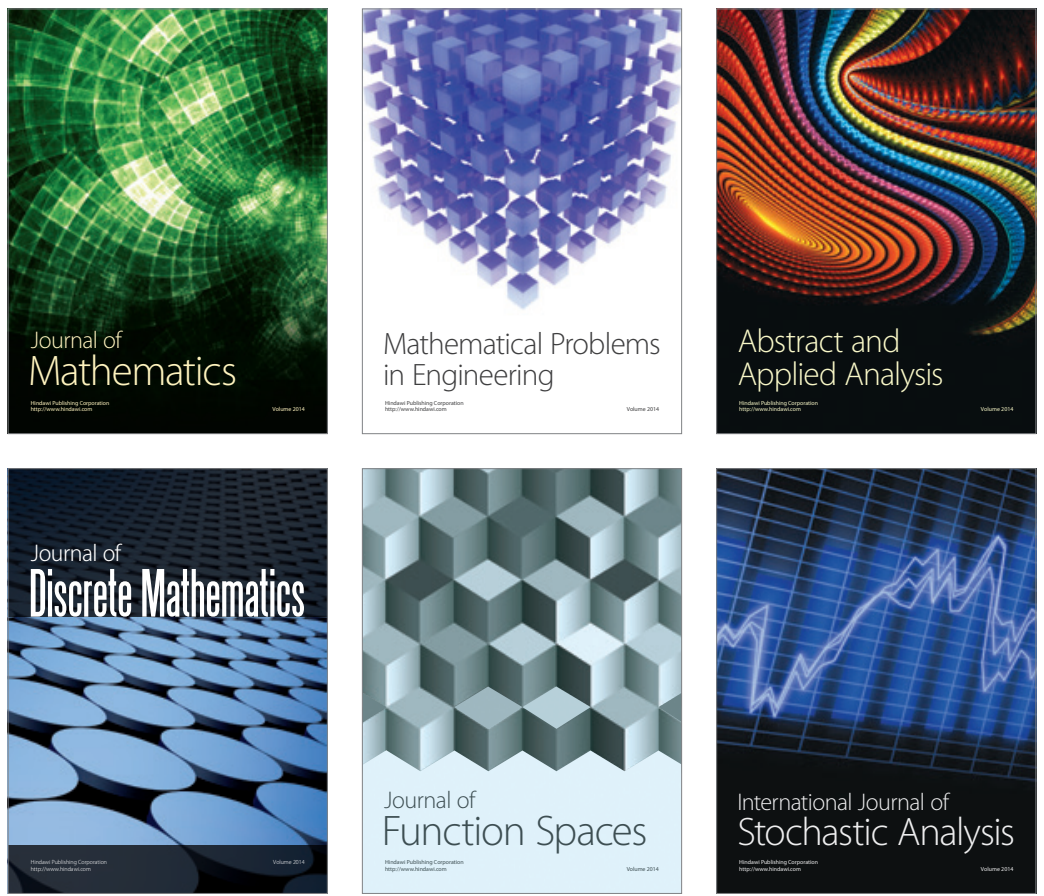

Journal of

Function Spaces

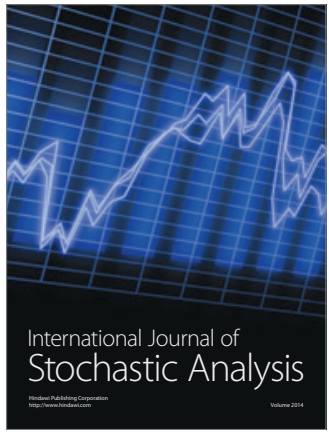

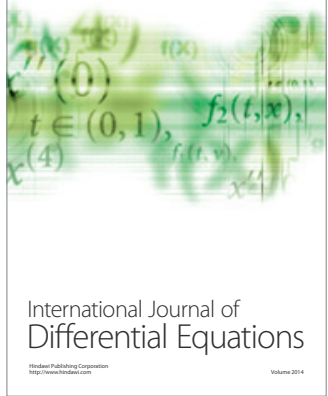
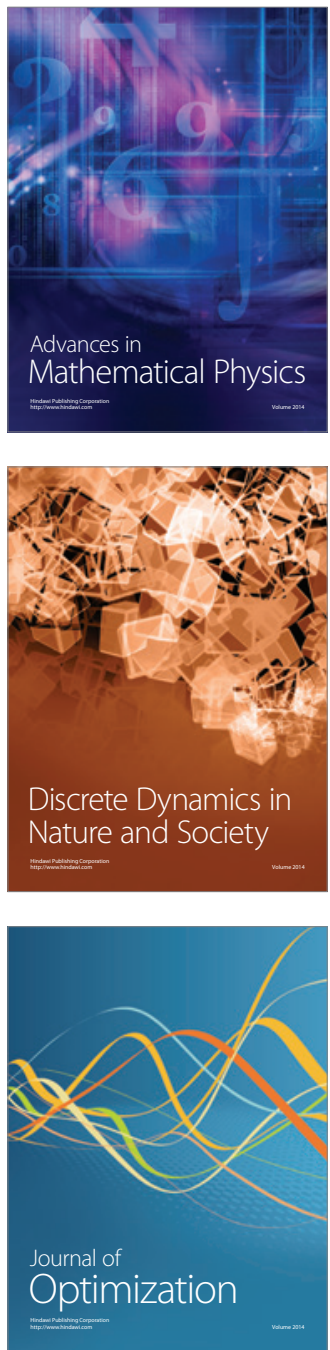